\title{
OCORRÊNCIA E ASPECTOS MACRO E MICROSCÓPICOS DO CISTO DE INCLUSÃO DA SEROSA UTERINA EM CADELAS E GATAS
}

\section{(Occurrence and macro and microscopic aspects of uterine serosal inclusion cysts in bitches and queens)}

\author{
Lucien Roberta Valente Miranda de Aguirra*, Eduardo Henrique Marsolla, Marcella \\ Katherine Marques Bernal, Sandy Kelly Souza Marques da Silva, Washington Luiz \\ Assunção Pereira
}

\begin{abstract}
*Correspondência: lucyaguirra@yahoo.com.br:
RESUMO: Objetivou-se relatar a ocorrência de cistos de inclusão da serosa uterina em cadelas e gatas, bem como descrever seus aspectos macroscópicos e microscópicos. Foram utilizados 200 úteros (100 cadelas e 100 gatas) procedentes de ovariosalpingohisterectomia eletiva. As peças foram fixadas inteiras em formaldeído a 10\% tamponado, seguindo-se ao processamento histológico de rotina, com as lâminas coradas pela hematoxilina e eosina. Os animais de estudo foram classificados segundo a espécie, a faixa etária, a parição e o uso de contraceptivos. Os dados obtidos foram analisados pelo teste Qui-quadrado, com nível de significância de $5 \%$. Dos úteros analisados $7 \%$ das cadelas e $1 \%$ das gatas apresentaram cistos de inclusão da serosa uterina, não havendo diferença estatística significativa para a faixa etária, a parição e o uso de contraceptivos interespécie. Macroscopicamente, foram observadas dilatações císticas únicas localizadas na região miometrial ou na superfície serosa do útero. Estes cistos estavam presentes na porção cranial ou medial dos cornos uterinos. Ao corte, apresentaram conteúdo líquido de aspecto seroso. Histologicamente, as dilatações císticas localizavam-se entre o miométrio e o perimétrio ou apenas na camada miometrial. O revestimento interno dos cistos era composto por epitélio variando em cúbico, colunar, pavimentoso, estratificado ou misto. Conclui-se que o cisto de inclusão da serosa uterina é uma alteração patológica com baixa incidência em cadelas e gatas, que causa irregularidade na superfície uterina, pela formação dos cistos, e apresenta ampla variedade de revestimento epitelial interno.

Palavras-chave: canina; cistos uterinos; felina; histopatológico
\end{abstract}

\begin{abstract}
The aimed to report the occurrence of uterine serosal inclusion cysts in bitches and queens, as well as describe their macroscopic and microscopic aspects. Were used 200 uteri (100 bitches and 100 queens) coming from elective ovariohysterectomy. The pieces were fixed in a solution of $10 \%$ buffered formaldehyde, followed by routine histologic processing, with slides stained with hematoxylin and eosin. The studied animals were classified according to species, age, parity and use of contraceptives. Data were statistically analyzed using Chi-square test with a significance level of $5 \%$. Of the uteri analyzed $7 \%$ Bitches and $1 \%$ of queens showed uterine serosal inclusion cysts, with no statistically significant difference interspecies for age, parity and use of contraceptives. Macroscopically, were observed single cystic dilations localized in myometrial region or on serosal surface of the uterus. When cut, observed a serous fluid content. Histologically, cystic dilations were located between the myometrium and perimetrium or just in myometrial layer. The internal liner of the cysts was composed of varying cubic epithelium, columnar, squamous, stratified or mixed. It was concluded that the uterine serosal inclusion cysts is a pathological change with low incidence in bitches and queens, causing irregularity in the uterine surfasse because the formation of cysts and has a wide range of internal epithelial lining..
\end{abstract}

Key Words: canine; uteri cystis; feline; histopathological 


\section{INTRODUÇÃO}

Os cistos de inclusão da serosa uterina apresentam literatura escassa e incidência pouco conhecida em cadelas e gatas, isto porque, essa alteração normalmente é encontrada de forma incidental durante $\mathrm{OSH}$ ou laparotomia (Johnston et al. 2001). Apesar de Ortega-Pacheco et al. (2007) terem relatado a ocorrência de 15 casos desses cistos após análise de 300 genitais de cadelas provenientes de OSH eletiva.

A etiopatogenia desses cistos é diversificada e pouco conhecida. É provável que um fluxo de sangue anormal para o órgão possa contribuir para a formação desses cistos, como resultado de drenagem insuficiente, devido ao retorno venoso deficiente e angiogênese das arteríolas (Schoon et al., 1999). Também são relatados a obstrução dos canais linfáticos a partir de um efeito gravitacional no útero durante a prenhez, o parto ou o pósparto (Kenney e Ganjam, 1975) e as contrações rápidas do miométrio durante a involução uterina no pós-parto (McEntee, 1990).

Morfologicamente, os cistos de inclusão da serosa uterina podem variar de alguns milímetros até vários centímetros de diâmetro (Kenney e Ganjam, 1975), sendo identificados em qualquer região do órgão, contudo, usualmente são observados na base de ambos os cornos uterinos ou nas suas proximidades, geralmente na camada miometrial (Eilts et al., 1995). Estes cistos são benignos e fisiologicamente inativos, não interferindo na função reprodutiva (Godfrey e Silkstone, 1998). Histologicamente, é observada uma dilatação cística, geralmente no miométrio exterior, revestida por camada única ou múltipla de células cuboidais ou escamosas. Em alguns casos estes cistos podem conter material eosinofílico ou amorfo (Godfrey e Silkstone, 1998).

Dessa forma, objetivou-se relatar a ocorrência de cistos de inclusão da serosa uterina em cadelas e gatas procedentes

de ovariosalpingohisterectomia eletiva, bem como descrever os aspectos macroscópicos e microscópicos.

\section{MATERIAL E MÉTODOS}

Foram utilizados 200 úteros, sendo 100 de cadelas e 100 de gatas, selecionados aleatoriamente através do projeto de extensão denominado "Vida Digna" da Universidade Federal Rural da Amazônia (UFRA), que realiza ovariosalpingohisterectomia (OSH) eletiva gratuita em caninos e felinos na faixa etária de seis meses a seis anos de idade e que apresentem estado de saúde aparente.

Os animais de estudo foram classificados segundo a faixa etária (animais $>6$ meses $a \leq 3$ anos $e$ animais $>3$ anos a $\leq 6$ anos), a parição (nulíparas e pluríparas) e o uso de contraceptivos (sim e não).

Os úteros procedentes de OSH foram obtidos diretamente na sala de cirurgia do bloco cirúrgico do Hospital Veterinário Profo Mário Teixeira (HOVET-UFRA), sendo encaminhados ao Laboratório de Patologia Animal (LABOPAT/UFRA), para realização de limpeza em água corrente, remoção do ligamento largo e fixação das peças.

As peças foram acomodadas e fixadas inteiras em recipiente de polietileno com tampa, contendo solução de formaldeído a $10 \%$ tamponado, por período de 48 horas. Posteriormente, foram selecionadas amostras através de cortes longitudinais de 3 a $5 \mathrm{~mm}$ de espessura das regiões que apresentaram os cistos de inclusão da serosa uterina. Em seguida, o material foi processado histológicamente segundo a técnica de 
rotina descrita por Tolosa et al. (2003), com as lâminas coradas pela Hematoxilina e Eosina (HE).

Os dados obtidos foram tabulados e analisados estatisticamente utilizando o teste Qui-quadrado, com auxílio do Pacote Estatístico Statistical Analysis System, SAS (1995). O nível de significância utilizado foi de $5 \%$ $(\mathrm{P}<0,05)$.

\section{RESULTADOS E DISCUSSÃO}

Dos úteros analisados 7\% (7/100) das cadelas e $1 \%$ (1/100) das gatas apresentaram cistos de inclusão da serosa uterina, havendo diferença estatística significativa entre as espécies. Esse resultado está de acordo com Godfrey e Silkstone (1998) que referiram uma infrequente ocorrência em cadelas e rara em gatas. Já OrtegaPacheco et al. (2007) observaram uma percentagem próxima a deste estudo, onde $5 \% \quad(15 / 300)$ das cadelas apresentaram cisto de inclusão da serosa uterina.

A faixa etária não representou um fator determinante na ocorrência dessa enfermidade em cadelas $(P>0,05)$, visto que, tanto animais abaixo quanto acima de três anos foram acometidos. A única gata acometida estava na faixa etária maior que seis meses e menor ou igual a três anos (Tabela 1). Neste contexto, não existem pesquisas que relatem uma predisposição ao desenvolvimento de cistos de inclusão da serosa uterina em cadelas e gatas, segundo a idade. Já tendo sido descrita em cadela de seis anos (Arnold et al. 1996) e gata com cinco anos (Godfrey e Silkstone, 1998).

Em relação ao número de partos, não houve diferença estatística significativa $(P>0,05)$ entre as cadelas nulíparas e pluríparas, enquanto a gata era plurípara (Tabela 1). Para alguns autores animais que já pariram são mais predispostos a desenvolverem cistos de inclusão da serosa uterina, já que essa alteração pode ser desenvolvida por obstrução dos canais linfáticos do útero durante a prenhez, parto ou pós-parto, ou ser causada por contrações rápidas do miométrio durante a involução uterina no pós-parto (Kenney e Garjam, 1975; McEntee, 1990). Os relatos observados na literatura também reforçam essa hipótese por serem descritos em cadela (Arnold et al. 1996) e gata (Godfrey e Silkstone, 1998) plurípara

\begin{tabular}{|c|c|c|c|c|}
\hline \multirow{3}{*}{$\begin{array}{l}\text { Variáveis }{ }^{*} \\
\text { Id ade }\end{array}$} & \multicolumn{4}{|c|}{ E spécies } \\
\hline & \multicolumn{2}{|c|}{ Canina } & \multicolumn{2}{|c|}{ Felina } \\
\hline & $\mathrm{n}^{1}$ & $\%$ & $\mathrm{n}^{1}$ & $\%$ \\
\hline $\begin{array}{l}>6 \mathrm{me}=3 \text { anos } \\
>3 \text { anos } \mathrm{e}=6 \text { anos }\end{array}$ & $\begin{array}{l}2 \\
5\end{array}$ & $\begin{array}{l}28,57 \\
71,43\end{array}$ & $\begin{array}{l}1 \\
0\end{array}$ & $\begin{array}{c}100,00 \\
00,00\end{array}$ \\
\hline Total & 7 & 100,00 & 1 & 100,00 \\
\hline \multicolumn{5}{|l|}{ Parição } \\
\hline Nuliparas & 3 & 42,86 & 0 & 00,00 \\
\hline Pluriparas & 4 & 57,14 & 1 & 100,00 \\
\hline Total & 7 & 100,00 & 1 & 100,00 \\
\hline \multicolumn{5}{|c|}{ Uso de contraceptivos } \\
\hline Não & 2 & 28,57 & 0 & 00,00 \\
\hline Sim & 5 & 71,43 & 1 & 100,00 \\
\hline Total & 7 & 100,00 & 1 & 100,00 \\
\hline
\end{tabular}

O uso de contraceptivos se fez presente em $71,43 \%$ das cadelas e a gata do estudo (Tabela 1), o que apesar de não representar uma distinção significativa $(P>0,05)$ entre os animais que usam ou não contraceptivos, possibilita indicar a participação dos hormônios sintéticos no desenvolvimento dos cistos de inclusão da serosa uterina.

Schlafer e Miller (2007) sugerem que a formação desses cistos possa estar associada à disfunção hormonal e, segundo Vural et al. (2004), um elevado nível de estrogênio no ciclo éstrico pode atuar como fator causal dessa alteração, aumentando as contrações do útero.

Macroscopicamente, foram observadas dilatações císticas únicas (Fig. 1A), localizadas na região miometrial (Fig. 1B) ou na superfície serosa (Fig. 2A). Em um caso, o cisto apresentou-se conectado a superfície serosa por um fino pedículo (Fig. 2B). Estes cistos estavam presentes na 
porção cranial dos cornos uterinos, próximo ao respectivo ovário e, em um caso, na porção medial do corno uterino. Ao corte, os cistosapresentaramconteúdo líquido de aspecto seroso.

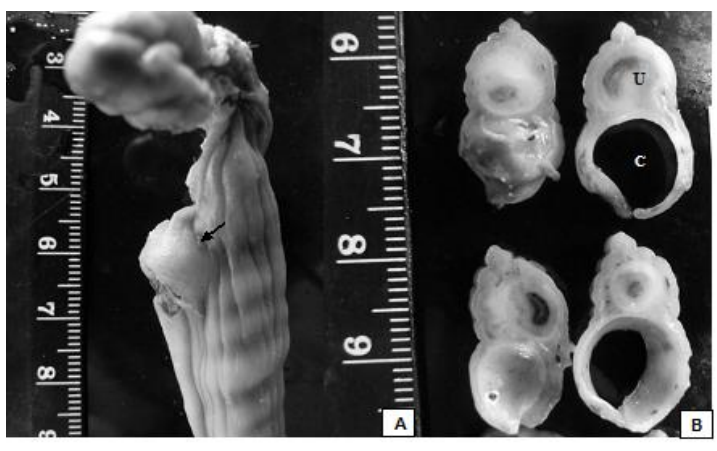

Figura 1 - Cisto de inclusão da serosa em útero de cadela. A. Dilatação cística única localizada na porção cranial do corno uterino (seta). B. Seção de cort
mostra dilatação cística (C) em região miometrial do corno utenino (U).

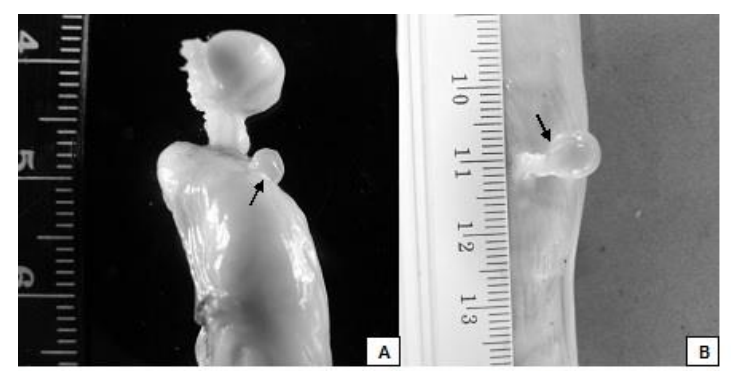

Figura 2 - A. Cisto de inclusão da serosa em útero de gata. Dilatação cística única na porção cranial do corno uterino (seta). B. Cisto de inclusão da serosa em pediculo (seta)

A descrição macroscópica para os cistos de inclusão da serosa uterina é semelhante ao referido por Mcentee (1990), embora o tipo multifocal não tenha sido observado neste estudo. Já Godfrey e Silkstone (1998) identificaram o tipo multiloculado e a presença de pedículo de ligação.

Kennedy e Miller (1993) relataram que esses cistos ocorrem geralmente como processo único, conforme observado no presente estudo, embora Kaiser (1978) tenha descrito a presença de múltiplos cistos na superfície serosa do útero de uma cadela.

Eilts et al. (1995) reportaram que os cistos de inclusão da serosa uterina ocorrem geralmente na camada miometrial, estando situados na base de ambos os cornos uterinos ou nas proximidades, o que discorda dos casos deste estudo visto que a maioria dos cistos ocorriam na região cranial do corno uterino.

Microscopicamente, foram observadas dilatações císticas únicas localizadas entre o miométrio circular e o perimétrio (Fig. 3A) ou apenas na camada miometrial circular (Fig. 3B). Em um caso o cisto promoveu deslocamento e perda da morfologia habitual entre 0 endométrio e 0 miométrio.

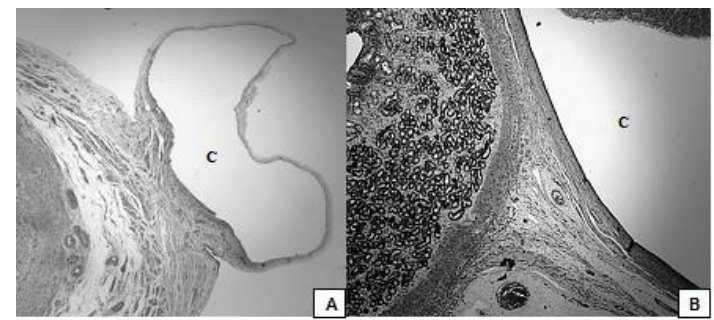

Figura 3 - A. Cisto de inclusão da serosa em útero de gata. Dilatação cística única (C) situada entre a camada miometrial circular e o perimétrio. HE, Obj. 4x. B Cisto de inclusão da serosa em útero de cadela. Dilatação cística (C) localizada no miométrio circular. HE, Obj. $20 \mathrm{x}$.

Os cistos eram revestidos internamente por epitélio variando em cúbico, colunar, pavimentoso ou estratificado e, em alguns casos, mais de um tipo epitelial estava presente no mesmo cisto

Os achados microscópicos estão de acordo com as descrições de Godfrey e Silkstone (1998) em gata e Sevimli et al. (2012) em búfala, onde esses últimos relataram ainda a presença de material homogêneo, amorfo e eosinofílico no interior dos cistos, fato não observado neste estudo

\section{CONCLUSÃO}

O cisto de inclusão da serosa uterina é uma alteração patológica com baixa incidência em cadelas e gatas, causando irregularidade na superfície uterina, pela formação dos cistos, que apresentam ampla variedade de revestimento epitelial interno. 


\section{REFERÊNCIAS}

ARNOLD, S.; HUBLER, M.; HAUSER, B. et al. Uterine serosal inclusion cysts in a bitch. Journal of Small Animal Practice, v. 37, p. 235-237, 1996.

EILTS, B.E.; SCHOLL, D.T.; PACCAMONTI, D.L. et al. Prevalence of endometrial cysts and their effect on fertility. Biology of Reproduction, v. 1, p. 527-532, 1995.

GODFREY, D.R.; SILKSTONE, M.A. Uterine serosal inclusion cysts in a cat. Veterinary Record, v. 142, p. 673, 1998.

JOHNSTON, S.D.; KUSTRITZ, M.V.R.; OLSON, P.N.S. Canine and Feline Theriogenology. Philadelphia: W.B. Saunders Company, 2001. $592 \mathrm{p}$.

KAISER, S. Uterine serosal inclusion cysts in a bitch. Kleinterpraxis, v. 23, p. 397, 1978.

KENNEDY, P.C.; MILLER, R.B. The female genital system. In: JUBB, K.V.F.; KENNEDY, P.C.; PALMER, A.C. Pathology of domestic animals. London: Academic Press, London, 1993. p. 349-470.

KENNEY, R.M.; GANJAM, V.K. Selected pathological changes of the mare uterus and ovary. Journal of Reproduction and Fertility, v. 23, p. 335-339, 1975.

MCENTEE, K. The ovary. In: MCENTEE, K. Reproductive Pathology of Domestic Animals. San Diego: Academic Press Inc, 1990. p. 31-51.

ORTEGA-PACHECO, A.; SEGURA-CORREA, J.C.; JIMENEZ-COELLO, M. et al. Reproductive patterns and reproductive pathologies of stray bitches in the tropics. Theriogenology, v. 67, p. 382-390, 2007.

SCHLAFER, D.H.; MILLER, R.B. Female genital system. In: JUBB, K.V.F.; KENNEDY, P.C.; PALMER, N. Pathology of domestic animals. Vol. 2. San Diego: Academic, 2007. p. 431-478.

SCHOON, D.; SCHOON, H.A.; KLUG, E. Angioses in the equine endometriumpathogenesis and clinical correlations. Pferdeheilkunde, v. 15, p. 15:541-546, 1999.

SEVIMLI, A.; OZENC, E.; ACAR, D.B. Oviduct cyst observed together with a uterine serosal inclusion cyst in the Anatolian water buffalo - a case report. Acta Veterinaria, v. 81, p. 235-237, 2012.

STATISTICAL ANALYSIS SYSTEM. SAS user's guide: statistics. Versão 5. Cary: SAS; 1995. 943 p.
TOLOSA, E.M.C.; BEHMER, A.O.; FREITASNETO, A.G. Manual de técnicas para histologia normal e patológica. Barueri - SP: Manole, 2003. $331 \mathrm{p}$.

VURAL, S.A.; HALIGUR, M.; OZENC, E. Uterine serosal inclusion cysts in dogs: Pathomorphological and immunohistochemical findings (in German). Kleintierpraxis, v. 49, p. 375-377, 2004. 